\title{
Intervención educativa y programa lúdico en el adulto mayor
}

\section{Educative intervention and a ludic program in the elderly adult}

\author{
Cruz Mayra*, Cruz Nube y Olivet Imilse \\ Universidad Católica de Cuenca \\ Cañar 030350, Ecuador \\ *maytecg1@ hotmail.com
}

\begin{abstract}
Resumen
Se realizó un estudio sobre el nivel ansiedad y estrés en los adultos mayores del Cantón Suscal centro con el objeto de intervenir con un programa educativo y lúdico para disminuir el nivel de ansiedad y estrés. Integrado por 72 adultos mayores de entre 65 a más de 90 años de edad e involucrando al personal del centro de salud para la continuación de actividades en beneficio de la población, a partir de este estudio se realizó encuestas a las personas involucrado, identificando de esta manera que el $90 \%$ de personas en el proceso de envejecimiento conlleva a múltiples problemas que causan al adulto mayor ansiedad y estrés, provocado por la soledad en un $36 \%$, las enfermedad en un $46 \%$ en un $11 \%$ la falta de ingresos; con estas estadísticas se programó una capacitación educativa sobre hábitos saludables en las distintas patologías, el autocuidado, prevención de caídas, y un taller recreativo con las necesidades de aprendizaje identificadas para minimizar las angustia que asiente al adulto. Se puede concluir que el programa de implementación educativa fue efectiva ya que el nivel de satisfacción y conocimiento incremento en el adulto mayor y se consiguió que el personal del centro de salud se involucre con la conformación del club de adultos mayores.
\end{abstract}

Palabras clave: Intervención educativa, programa lúdico, adulto mayor.

\begin{abstract}
A study was made about the anxiety level and stress in elderly adults in the downtown in Suscal canton in order to intervene with an educational and entertaining program to reduce the level of anxiety and stress. It was made up by 72 adults aged from 65 to 90 years old and involving the health center staff to continue developing activities for the benefit of the population, there were made surveys applied to the involved people based on this study, so it was identified that $90 \%$ of the elderly people in the process of getting order will carry several problems that cause anxiousness and stress in the elderly adult, $30 \%$ was caused by loneliness, $46 \%$ due to sicknesses, $11 \%$ lack of incomes; stress caused by loneliness a $36 \%$, of the disease $46 \% 11 \%$ lack of income; taking into consideration these statistics, an educational training on healthy habits in different diseases in different pathologies was programmed, the self-care, fall prevention, and a recreational workshop with identified learning needs to minimize the torment the adult. It can be concluded that the implementation of the educational program was effective because the level of satisfaction and knowledge increase in the elderly and it was attained that the health center staff get involved with the elderly adult club confirmation.
\end{abstract}

Key words: Educative intervention and a ludic program in the elderly adult.

\section{INTRODUCCIÓN}

Partiendo de una base conceptual, el adulto mayor pasa por una etapa de la vida que se considera como la última, en la que los proyectos de vida ya se han consumado, siendo posible poder disfrutar de lo que queda de vida con mayor tranquilidad $^{1}$. Usualmente las personas de la tercera edad han dejado de trabajar, o bien jubilan, por lo que su nivel de ingresos decrece en forma considerable, lo que junto con los problemas de salud asociados a la edad pueden traer consecuencias en todos los ámbitos de su vida.

El estrés a cualquier edad se considera un factor de riesgo para la salud, aún más a personas vulnerables como son las de la tercera edad; el estrés es una sobre tensión que genera un estado de desequilibrio en el ser humano, la ansiedad en si es un fenómeno en una emoción normal del ser humano necesaria para garantizar la supervivencia ante un peligro potencial sin importar el rango etario, cuando este eleva su grado de intensidad y duración se vuelve patológico, en la edad adulta mayor se puede establecer como un grado de ansiedad generalizada.

El paso de día a día conlleva a que en la vida exista un nivel de estrés elevado ya sea por, las nuevas tecnologías, la política gubernamental, el correr diario, son factores que intervienen en este tipo de emociones, estudios realizados y presentados en un artículo de expresan que En lo que 
respecta a las fuentes de estrés, quienes realizaron distintos estudios sobre las fuentes de estrés en los adultos mayores, concluyendo que la muerte de una persona significativa, la propia enfermedad, y el retiro de la actividad laboral son experiencias muy frecuentes y estresantes en la vejez. Plantean también, que existen problemáticas específicas de la vejez como ser:

- La modificación de los contactos sociales.

- La disminución de los contactos sociales.

- El aislamiento social ${ }^{2}$.

Con lo antes citado y el estudio realizado para este artículo no se encuentra lejano a la realidad ya que se enfoca a los adultos mayores del Cantón Suscal de la Provincia de Cañar, la vulnerabilidad de esta enfermedad se hace presente por las diferentes causas y factores que atribuyen a que exista una sobretensión del estado de equilibrio de la persona y por ende el estado emocional se altere ${ }^{3}$, ante esto se puede expresar la soledad, las enfermedades, el sustento económico ya que las funciones de trabajo se suspenden y la percepción de un suelo es mínima que en ocasiones no sustenta un buen aporte para satisfacer las necesidades humanas, siendo de esta manera un eje importante la realización del programa educativo en el que los adultos mayores tengan mejores conocimientos sobre las principales causas que provoca este desequilibrio con este programa se ha podido lograr que los adultos mayores conlleven un estilo de vida saludable, como un proceso social conducta o comportamiento del individuo, como una alimentación acorde a sus necesidades, con actividades recreativas, para así disminuir el nivel de estrés y ansiedad y alcanzar el bienestar y vida en estas personas ${ }^{4}$.

La conformación del club de adultos mayores mejoro la calidad de vida ante la sociedad ya que pueden descubrir y transmitir sus experiencias, facilitar el dialogo, en un grupo que favorezca sus expectativas.

\section{Marco Teórico}

\subsection{Concepto de Adulto Mayor(AM)}

Se considera un AM a la persona que envejece como un proceso gradual, natural e inevitable, son fases que que se dan cambios a nivel biológico, corporal, psicológico y social. Transcurre en el tiempo y esta delimitado por éste ${ }^{6}$ se llama adulto Mayor a los que cursan por el proceso del envejecimiento y que cronológicamente pueden ubicarse entre los 55/60 años (entroncando con lo que se llama la edad media de la vida) y los 79/80 años. A partir de los 80 años tomamos la vejez y por tanto llamamos viejos a los que la transitan ${ }^{7}$.

Existen varios criterios para decir que una persona es vieja:

Criterio cronológico edad en años; relacionado con el retiro o jubilación obligatoria.
Criterio Físico Se toman en cuenta los cambios físicos producidos en la persona, como cambios en la postura, forma de caminar, facciones, color del pelo, piel, capacidad visual y auditiva, disminución en la capacidad del almacenamiento de la memoria que afecta a los tres tipos de memoria (a corto, mediano y largo plazo) de forma directa, sueño alterado, etc. Todo esto relacionado a la alteración en forma notable de las actividades cotidianas $^{7}$.

Criterio Social Según el papel o rol que desempeñan en la sociedad.

\subsection{Factores que aceleran el envejecimiento}

- Alimentación excesiva.

- Stress.

- Hipertensión.

- Tabaquismo y alcoholismo.

- Obesidad.

- Soledad, poca participación socio laboral.

- Sedentarismo.

- Poca actividad física.

Con la edad disminuye la frecuencia cardíaca máxima y el consumo máximo de oxigeno, declinación que comienza a partir de los 30 años; esta declinación es mas rápida con la inactividad o el abandono del entrenamiento ${ }^{8}$.

De todo lo expuesto anteriormente podemos sintetizar que AM tienen los siguientes problemas que determinan esta investigación que es la intervención educativa y programa en el AM

- El problema biológico: enfermedades crónicas agregadas y el proceso normal de involución.

- El problema financiero. El problema del mejor aprovechamiento vital(cómo administrar el tiempo que queda de vida).

- El problema sexual. El deseo continua, pero desarrollo disminuye.

- El problema filosófico: angustia de muerte y el que pasara después de la muerte.

- Las relaciones sociales: disminuyen el círculo social se restringe

- El problema lúdico: este es un punto importante ya que los individuos no saben como ocupar el tiempo libre que antes ocupaban con el trabajo; no saben cómo recrearse, ni que actividades físicas realizar.

\subsection{Actividades en los adultos mayores}

Existen diferentes tipos de actividades que desempeñan los ancianos en el tiempo libre entre los cuales son numerosos dependiendo del estado de salud, la capacidad de moverse o sus limitaciones y sobre todo las preferencias personales del individuo. Algunas de las actividades más populares que un adulto mayor puede realizar para ocupar su el tiempo libre son practicar la jardinería o la lectura, 
ver la televisión, observar acontecimientos deportivos, participar en actividades sociales, visitar amigos y familiares, pasear o caminar, y poner interés en sucesos educacionales y creativos ${ }^{9}$.

Estas actividades pueden ser complementadas por las actividades propuestas en el modelo de envejecimiento exitoso de Rowe y Kahn ${ }^{10}$ en sus tres componentes, que son:

1) Minimización de riesgos para la salud por medio de actividades de protección a la salud

2) Maximización de capacidades físicas y cognitivas a través de la realización de AIVD.

3) Participación activa en actividades sociales y actividades productivas que no necesariamente generen una remuneración económica ${ }^{9}$.

\section{Materiales y métodos}

Se ha realizo un estudio descriptivo, analítico y prospectivo con orientación cuantitativa mediante encuesta y evidencia documental que fue aplicada a 72 adultos mayores, resultado del $60 \%$ del universo total, escogidos al azar; de entre los 65 años a más de 90 años, el universo o población constituye de 120 personas que son consideradas como adultos mayores.

La encuesta describió preguntas relacionadas acerca de los datos generales entre ellas se identificó po grupo etario entre los que en un $58 \%$ se encontraban entre edades de 65 - 75 años, $32 \%$ de 76-85, 86 a más de 90 años en un $10 \%$, en cuanto al sexo se puede establecer que el $63 \%$ comprende al sexo femenino y el $37 \%$ al sexo masculino, a más de esto se encuesto sobre él tipo de enfermedades que padece, sobre el conocimiento de estilo de vida, que es lo que más le hacía sentir triste o con ansiedad, entre otras, se analizó e interpreto los datos.

Los datos obtenidos fueron tabulados en hojas de cálculo Excel y se trabajó con la distribución de frecuencias y el cálculo porcentual de la estadística descriptiva para el análisis e interpretación de los resultados. Dentro de los métodos teóricos se utilizaron el histórico lógico, la inducción-deducción y el análisis y síntesis. Posteriormente luego de la interpretaciòn de datos, se elaboró un programa educativo y recreativo, con el fin de disminuir el nivel de estrés y ansiedad de los adultos mayores. Las temáticas fueron programadas utilizando estrategias de conferencias, y actividades recreativas, entre ello tenemos, dinámicas, bailoterapia ejercicios de relajación, se conformó el club de adultos mayores, comprometido el Centro de salud para su consecución

\section{Resultados}

De acuerdo a los datos obtenidos los adultos mayores en su mayor porcentaje que es del $42 \%$ se dedican a los QQ.DD, $17 \%$ son comerciantes ,8\% agricultores y un $26 \%$
Factores que intervienen en el bienestar del AM

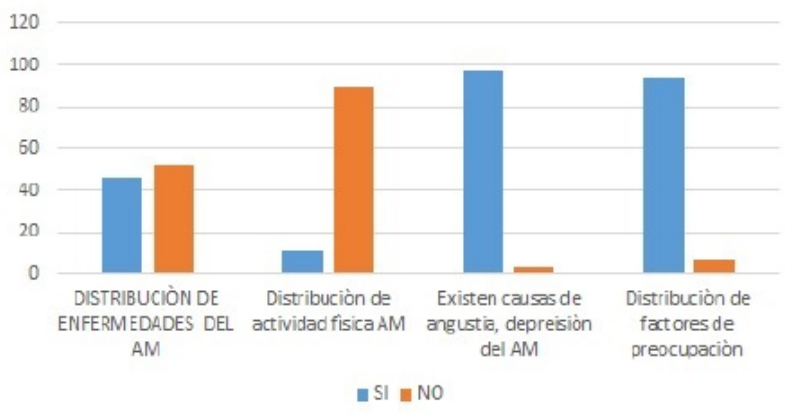

Tabla 1. Estadística de factores que intervienen en el bienestar AM.

no cumplen actividad alguna, motivo por el cual es causa de preocupación y ansiedad por estar inactivo, a esto se le suma la actividad económica que maneja cada adulto mayor, la dedicación es el comercio en un $21 \%$, remesas de migrantes en un $14 \%$, agricultura en un $14 \%$, los que reciben el bono de desarrollo en un $39 \%$, los que reciben lo pensión por jubilación y los que viven en arriendos se representa en un porcentaje mínimo esta preocupación aumenta con la angustia de que el bono les sea retirado.

En la distribución sobre los estilos de vida que deben llevar desconocen en $78 \%$ y el $22 \%$ sabe de lo que se trata, aplicando el programa educativo incremento notablemente en $75 \%$ conoce el tema y un $25 \%$ no conoce.

Realizando un análisis exhaustivo el adulto mayor padece enfermedades en un mayor porcentaje en un $95 \%$, la importancia de este tema se expone a que las enfermedades conllevan a angustia a las personas y complicaciones de las mismas por lo que intereso estudiar más a fondo sobre los sistemas de vida que llevan, alimentación, entorno geográfico para de esta manera brindar una educación adecuada.

En la tabla 1 se observa que existen en su totalidad $89 \%$ de adulto mayores no se ejercitan conllevando a adquirir con mayor facilidad enfermedades, también se observa que existen causas para la angustia y depresión en un $97 \%$ total, sumando en un $46 \%$ por enfermedad más el $36 \%$ de soledad, un $11 \%$, y muerte de personas cercanas en $4 \%$.

Los factores que intervienen para el estrés AM son diferentes entre ellas se tiene preocupaciones cansancio falta de ejercicio sumando un $97 \%$

\subsection{Discusión}

La salud y bienestar de las personas son el componente más importante que conserva un individuo para la supervivencia, llevando un estilo de vida saludable, para ello los individuos deben conocer e incorporar a su vida con el objetivo de preservar su salud y contribuir a protegerla. 
Durante la realización de trabajo se puso énfasis en el desarrollo del programa educativo con la participación de los adultos mayores y personal de centro de salud comprometiéndoles con su asistencia, para la conformación del club de adultos mayores.

La falta de conocimiento de los buenos estilos de vida y la falta de recreación en los adultos mayores fue evidenciada con su desconocimiento al aplicar las encuestas.

$\mathrm{El}$ abandono de ejercicios y las diferentes actividades lúdicas en el adulto mayor son causas predisponentes, al aumento de estrés y esto conlleva a agravar enfermedades cardiovasculares con las que ya padecen.

\section{Conclusiones}

- De acuerdo a los resultados obtenidos, los adultos mayores han disminuido con el pasar del tiempo en realizar actividad física como es la caminata y otras actividades físicas, por tal motivo la importancia de establecer un programa lúdico.

- La ansiedad y estrés se da por diferentes factores, en el adulto Mayor del Cantón Suscal centro atreves del trabajo de campo implementado la ansiedad y el estrés se da más por presentar diferentes enfermedades, y también por la soledad en pareja en la cual ellos se sienten muy tristes y lo que genera esta terrible enfermedad social.

- En el cantón Suscal, luego de la recolección de información y trabajo de campo realizado, hemos concluido que, a más de no contar con centros de apoyo al adulto mayor en lo que tienen que ver con la atención a patologías generadas por el estrés y la ansiedad, ellos acuden más con frecuencia al centro de salud y al seguro campesino del mismo cantón para chequeos, recibir medicación para el control de las diferentes enfermedades como son la presión alta y la diabetes.

- Con la elaboración de un programa educativo y recreativo, disminuye el nivel de estrés y ansiedad de los adultos mayores.

- Las temáticas fueron programadas utilizando estrategias de conferencias, y actividades recreativas, entre ello tenemos, dinámicas, bailoterapia ejercicios de relajación.
- La conformación del club de adultos mayores ayuda a que tengan un centro de distracción y por nde decremento el nivel de estrés

- Centro de salud del canton Suscal queda comprometido a velar por el bienestar de los AM y la consecución del club

\section{Referencias}

1. Mateus,YP. Cuidado adulto mayor http: //blogyulycitamateus.weebly.com/. [2010].p 1

2. Clemente A, Feldberg C, Tartaglin MF,Estefani D. Geriatría clínica http://www.geriatriaclinica.com. [2009].p 1

3. Bastidas, MB. Métodos de ansiedad y fobia normalidad o patología Boletín de novedades de psicología onlin.[2011] http://www.psicologia-online.com/infantil/ miedos-ansiedad-y-fobias.shtml

4. Campos PJ, Borja RM, Suarez BA, Arreo DV, Franco $\mathrm{DE}$, AsoVJ, Et al transtornos de la ansiedad. Amir enfermeria. Madrid-España [2013]

5. Wang, J.L., Schmitz, N., Dewa, C. y Stansfeld, S. Changes in perceived job strain and the risk of major depression: results from a population-based longitudinal study American Journal of Epidemiology, [2009] 169(9), 1085- 1091

6. DE ROSNAY, J. Qué es la vida Biblioteca Científica Salvat. Barcelona[1988].p 1

7. Moreno González, A. ncidencia de la Actividad Física en el adulto mayor. Revista internacional de medicina y ciencias de la actividad física y el deporte, 5(19), 222237.[2005]

8. CRESS, M., SMITH, E., THOMAS, D., JOHNSON, J. Effect of training on VO2máx., thigh strenght, and muscle morphology in Septuagenarian women. Med. And Sci. in Sport and Exercise. [1991]

9. Acosta QC, Gonzales A, Celis R. Psicologia y salud. Actividades de la vida diaria en el adulto mayor http://revistas.uv.mx/index.php/psicysalud/article/ view/639/1116. [2009]

10. Rowe, J.W. y Khan Human aging: usual and successful Science, 237,143- 149. [1987]

Recibido: 1 de junio de 2016

Aceptado: 16 de mayo de 2017

Cruz, Mayra : Licenciada en enfermería. MSC. Gerencia de la salud para el desarrollo Local. Profesora de carrera de enfermería. Universidad católica de Cuenca. Sede Cañar. Ecuador 
Cruz, Nube: Ing. en Electrónica y Telecomunicaciones, MSC en Seguridad Telemática. Gerente de de CGXS.CIA.LTDA. Contratista de CNT, Santo Domingo de los Sachilas. Docente de informatica y electrónica por 5 años en la Univ. José Peralta y el Tec. José Peralta. Correo electrónico:nube5502@gmail.com

Olivet, Imilse: Licenciada en enfermería. MSC. Longevidad satisfactoria, miembro titular de la SOCUENF. Profesora de carrera de enfermería. Universidad católica de Cuenca. Sede Cañar. Ecuador. Correo electrónico: iolivetl@ucacue.edu.ec 
